\title{
Estimación de la disposición a pagar por un sistema de recolección mejorado de residuos sólidos domésticos en la ciudad de Juliaca - 2020
}

\author{
Estimation of the availability to pay for the collection system of Domestic Solid \\ Waste in the city of Juliaca - 2020
}

\author{
"Julio Cesar Quispe Mamani \\ Marcial Guevara Mamani \\ ${ }^{2}$ Victor Rogelio Marca Maquera \\ Vladimir Ylich Felipe Mamani Sonco \\ ${ }^{3}$ Hugo Ruben Marca Maquera
}

\author{
ORCID: 0000-0002-3938-1459 \\ ORCID: 0000-0003-3545-1306 \\ ORCID: 0000-0002-8761-8000
}

\section{RESUMEN}

El servicio del sistema de recolección de los residuos sólidos domésticos de la ciudad de Juliaca-Perú requiere de un mejoramiento y planificación más eficientes que permitan superar la excesiva generación y acumulación de los residuos sólidos que se encuentran en basureros, botaderos y a la intemperie. Debido a que la inadecuada selección de residuos orgánicos e inorgánicos y su disposición final generan descomposición que causa malos olores y lixiviados, propiciando la proliferación de insectos y roedores, vectores de microorganismos patógenos de humanos y animales, causando así un impacto negativo en el medio ambiente. El objetivo de esta investigación fue estimar el valor económico que la población está dispuesta a pagar para mejorar el sistema de recojo de residuos sólidos domésticos. Se aplicó el método de valoración contingente y el modelo econométrico binomial Probit. Se realizaron encuestas a 382 hogares. Se concluye que el $51.75 \%$ de los encuestados están dispuestos a pagar por un sistema de recojo de residuos sólidos domésticos mejorado, con un costo promedio de S/ 5.36 mensuales; mientras que, el $41.10 \%$ de los encuestados no estaría dispuesto a pagar. Asimismo, los factores que influyen en dicha decisión fueron el nivel educativo, la ética ambiental y la conciencia ambiental de la población de Juliaca.

Palabra clave: Disponibilidad a pagar, método de valoración contingente, Modelo Probit, residuos sólidos.

\section{ABSTRACT}

The service of the collection system for domestic solid waste in the city of Juliaca-Peru requires improvement and more efficient planning to overcome the excessive generation and accumulation of solid waste found in landfills, dumps and open pit, all Once the non-proper selection of organic and inorganic waste and its final disposal generate a decomposition that causes bad odors and leachates, promoting the proliferation of insects and rodents that are vectors of pathogenic microorganisms in humans and animals and consequently causes a negative impact on the environment. The objective of the research work was to estimate the economic value that the population is willing to pay to improve the system for collecting domestic solid waste. The contingent valuation method and the Probit binomial econometric model were applied, where 382 households were surveyed. It is concluded that $51.75 \%$ of the respondents of the Juliaca population are willing to pay for an improvement in the system of collecting solid domestic waste with an average amount of S / 5.36 per month; while $41.10 \%$ of those surveyed would not be willing to pay, since said measure is the responsibility of the municipality; Furthermore, they do not have sufficient financial resources to pay. Likewise, the factors that influence said decision were the educational level they have, the environmental ethics and the environmental awareness of the Juliaca population.

Keywords: Availability to pay, contingent valuation method, Probit Model, solid waste.

${ }^{1}$ Escuela Profesional de Ingeniería Económica. Universidad Nacional del Altiplano. Puno - Perú.

${ }^{2}$ Escuela Profesional de Gestión Pública y Desarrollo Social. Universidad Nacional de Juliaca. Juliaca - Perú

${ }^{3}$ Escuela Profesional de Gestión Pública y Desarrollo Social. Universidad Nacional de Moquegua. Moquegua - Perú.

*Autor de correspondencia: jcesarqm@yahoo.es

Presentado: 02/04/20, Aprobado: 30/05/2020 


\section{INTRODUCCIÓN}

El manejo de los residuos sólidos (RS) es uno de los problemas más comunes en las ciudades de América Latina y del Perú. Su inadecuada recolección y disposición final contaminan la tierra, el agua y el aire (Mamani, 2019), ocasionando riesgos para la salud humana (Tudela, Martínez, Valdivia, Portillo \& Romo, 2009). La ciudad de Juliaca, según el Instituto Nacional de Estadística e Informática (INEI), es la decimotercera ciudad más poblada del Perú, con una población de 228726 habitantes, es conocida como la capital de la integración andina y forma parte de los cuatro distritos de la provincia de San Román (Puno) al sudeste de Perú (Huamaní et al., 2020). Registra una tasa de crecimiento poblacional de $2.9 \%$, ocupando una superficie de $533.5 \mathrm{~m}^{2}$ aproximadamente, situada a 3824 m s.n.m. al noroeste del Lago Titicaca (Huamán, 2019).

La ciudad de Juliaca se caracteriza por el desarrollo predominante de la actividad comercial, donde gracias a la conexión vial del transporte terrestre y aéreo se facilita el intercambio comercial a nivel regional, interregional y nacional. Junto a este desarrollo comercial se suma un crecimiento urbano muy desordenado (Armijo de Vega et al., 2012). Además de la generación de RS de manera descontrolada, existen serios problemas de recolección y almacenamiento en los espacios públicos (Pérez, 2015). La capacidad operacional de los servicios de recolección a los domicilios es deficiente, limitada e insuficiente para una población grande y creciente (Azqueta, 1994).

La ciudad de Juliaca no cuenta con un plan de gestión ambiental implementado de manera eficiente que pueda ayudar a superar las deficiencias en la acumulación de los residuos y su reaprovechamiento de la misma (Valdivia, et al., 2012). Bajo el esquema actual del sistema de gestión de residuos sólidos, esto se hace difícil porque el costo de la disposición de los RS no muestra completamente el costo ambiental que ocasiona (Vargas \& Cortés, 2018). Por lo que, para alcanzar una eficiencia social, es necesario que las familias valoren los costos que acarrea la disposición final, y así eviten tomar decisiones individualistas (Mamani, 2019). Actualmente, los productores y consumidores que participan en el proceso de la gestión de los RS no lo hacen de una manera integrada ni colectiva (Ibarrarán \& Cuevas, 2003).

Complementariamente, el sistema de recojo de residuos sólidos es limitado, siendo su situación crítica en los últimos años (Mamani, 2019). La valoración del entorno significa poder contar con un indicador de importancia en el bienestar de la sociedad a partir de las valoraciones individuales (Vargas \& Cortés, 2018). Además, es frecuente ver residuos sólidos y plásticos acumulados en basureros o botaderos a la intemperie (Mamani, 2019). Los botaderos de residuos sólidos impactan negativamente en el ambiente. Asimismo, mezclados con residuos orgánicos e inorgánicos generan descomposición que causa malos olores, lixiviados y propicia la proliferación de insectos y roedores, vectores de microorganismos patógenos de humanos y animales (Huamani, 2017). En síntesis, se tiene una limitada gestión de residuos por no contar con el financiamiento necesario para implementar un buen sistema ante esta problemática (Mamani, 2019). Por lo cual, se hace necesario conocer cuál es el importe monetario que pagaría la población para implementar un sistema mejorado (Azqueta, 1994).

Por ello, lo que se buscó con la presente investigación es responder la siguiente pregunta: ¿Cuál es la disponibilidad a pagar de la población para mejorar el sistema de recojo de los residuos sólidos domésticos, en la ciudad de Juliaca? Teniendo en cuenta que, con el crecimiento demográfico y el inadecuado manejo de los residuos sólidos en la ciudad de Juliaca y su disposición final (Rodriguez, 2017), se viene generando un deficiente sistema de recojo de residuos sólidos, contribuyendo hacia una mayor acumulación de los residuos sólidos en los espacios públicos (Guzmán \& Macías, 2012). Lo cual, a su vez, viene incrementando el nivel de riesgo sanitario y la propagación de plagas, ocasionando casos de enfermedades dérmicas $(86.66 \%)$, oculares $(73.33 \%)$, respiratorias $(96.6 \%)$, digestivas $(93.33 \%)$ y neuropsíquica $(87.5 \%)$ en personas que tienen contacto directo con los RS, evidenciándose un alto nivel de morbilidad y en consecuencia un bajo nivel de calidad de vida (Quilla, 2017).

Los objetivos fueron:

1. Estimar el valor económico que la población está dispuesta a pagar para mejorar el sistema de 
recojo de residuos sólidos domésticos en la ciudad de Juliaca.

2. Determinar los factores que influyen en la disponibilidad a pagar por un sistema de residuos sólidos domésticos.

3. Determinar la aproximación del nivel de ingresos de la población de la ciudad de Juliaca y determinar el nivel educativo de la población de la ciudad de Juliaca.

\section{MATERIALY MÉTODOS}

\section{Tipo y diseño de investigación}

El método utilizado en la presente investigación fue de tipo descriptivo y correlacional (Fernández et al., 2006).

\section{Población y muestra}

La población objeto de estudio asciende a 228726 personas, distribuidas en 67755 hogares (Huamaní et al., 2020). Se utilizó el método de valoración contingente con el modelo econométrico Probit, considerando un tipo de muestra probabilístico, específicamente aleatorio simple con una población finita, determinando el tamaño muestral de 382 hogares. La encuesta se aplicó a todo integrante que se haya encontrado en el proceso de aplicación y que sea mayor de 18 años, a fin de determinar la diversidad y la importancia que le dan los encuestados a la conservación del medio ambiente de acuerdo a sus edades. La muestra fue calculada con un nivel de confianza de $95 \%$ (Lacort, 2014).

\section{Instrumento de colecta y análisis de datos}

Se utilizó la encuesta, con preguntas relacionadas a las variables en análisis, ya sean dependientes o independientes, a fin de determinar las influyentes en la decisión de acceder al pago monetario por la mejora en el sistema de recolección de los residuos sólidos domésticos (Lacort, 2014). La información secundaria utilizada se obtuvo de la base de datos del Censo 2017, del Instituto Nacional de Estadística e Informática (INEI) y del Ministerio del Ambiente (MINAM).

Una vez obtenidos los resultados de las encuestas, se realizó una base de datos en el programa SPSS 14.0, para en análisis estadístico. Se realizaron las pruebas y los test para comprobar el grado de correlación que existe entre las variables (Valdivia et al., 2012). Para analizar la información se estimaron los modelos para la variable dependiente discreta, es decir, se estimó la probabilidad de una respuesta afirmativa, dados algunos parámetros (Girón, 2017). El modelo utilizado para estimar la probabilidad de respuesta afirmativa fue el Probit (Girón, 2017).

\section{Modelo Econométrico}

En la presente investigación se hizo un análisis de regresión binaria para ver la dependencia de la DAP de un adecuado sistema de recolección de residuos sólidos domésticos, en función de variables explicativas (Valdivia et al., 2012).

El Modelo Econométrico a estimar fue el siguiente:

$$
\begin{aligned}
P\left(\text { disp }_{\text {pagar }}\right) & =\beta_{0}+\beta_{1} \text { ingreso }_{\log }+\beta_{2} \text { edad }+\beta_{3} \text { educación }+\beta_{4} \text { Gen }+\beta_{5} E a+\beta_{6} N+\beta_{7} \text { evit }_{\text {contam }} \\
& +\mu
\end{aligned}
$$

Donde la variable dependiente binaria DAP simboliza si la persona está dispuesta a pagar por un adecuado sistema de recolección de residuos sólidos domésticos, que explica la valoración ambiental de la población en estudio (Valdivia et al, 2012). Esta variable depende del ingreso per cápita del hogar, nivel educativo, género, ética ambiental, número de integrantes en la familia y la conciencia ambiental (Huacani y Mamani, 2016).

\section{Variables a considerar en la Estimación Econométrica}

Las variables que se utilizaron para la estimación econométrica fueron los siguientes. Disponibilidad a

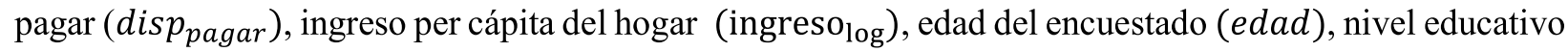
(educación), género $(G e n)$, ética ambiental $(E a)$, número de integrantes del hogar $(N)$ y conciencia ambiental (evit_contam). En la tabla 1 se hace la operacionalización de las variables utilizadas para la estimación del modelo (Girón, 2017). 
Estimación de la disposición a pagar por un sistema de recolección mejorado de residuos sólidos domésticos en la ciudad de Juliaca - 2020

Tabla 1. Características de las variables evaluadas para la estimación del modelo Probit

\begin{tabular}{|c|c|c|c|c|}
\hline Tipo de Variable & Variable & Notación & Concepto & Caracterización \\
\hline Dependiente & $\begin{array}{l}\text { Disponibilidad a } \\
\text { pagar }\end{array}$ & disp pagar & $\begin{array}{l}\text { ¿El encuestado está dispuesto a } \\
\text { pagar por el sistema de } \\
\text { recolección de residuos? }\end{array}$ & $\begin{array}{l}\text { Dicotómica: } \\
1=\text { Sí } \\
0=\text { No }\end{array}$ \\
\hline \multirow[t]{7}{*}{ Independiente } & $\begin{array}{l}\text { Ingreso per cápita } \\
\text { del hogar }\end{array}$ & ingreso $_{\log }$ & $\begin{array}{l}\text { Ingreso mensual promedio en el } \\
\text { hogar del encuestado }\end{array}$ & Continua y cuantitativa \\
\hline & $\begin{array}{ll}\begin{array}{l}\text { Edad } \\
\text { encuestado }\end{array} & \text { del } \\
\end{array}$ & edad & Edad de la persona encuestada & Continua y cuantitativa \\
\hline & Nivel educativo & educación & $\begin{array}{llll}\begin{array}{l}\text { Años de } \\
\text { encuestado }\end{array} & \text { educación } & \text { del } \\
\end{array}$ & Continua y cuantitativa \\
\hline & Género & Gen & Género del encuestado & $\begin{array}{l}\text { Dicotómica } \\
1=\text { Masculino } \\
0=\text { Femenino }\end{array}$ \\
\hline & Ética ambiental & $E a$ & $\begin{array}{l}\text { ¿El encuestado considera } \\
\text { importante la conservación del } \\
\text { medio ambiente? }\end{array}$ & $\begin{array}{l}\text { Dicotómica } \\
1=\text { Sí } \\
0=\text { No }\end{array}$ \\
\hline & $\begin{array}{lc}\text { Número } & d e \\
\text { integrantes } & \text { del } \\
\text { hogar } & \\
\end{array}$ & $N$ & $\begin{array}{l}\begin{array}{l}\text { Número de } \\
\text { conforman ersonas }\end{array} \\
\begin{array}{l}\text { encuestado } \\
\text { encue }\end{array}\end{array}$ & $\begin{array}{l}\text { Cuantitativa } \\
\text { cualitativa }\end{array}$ \\
\hline & $\begin{array}{l}\text { Consciencia } \\
\text { ambiental }\end{array}$ & evit_contam & $\begin{array}{l}\text { ¿El encuestado hace algo para } \\
\text { evitar la contaminación del } \\
\text { medio ambiente? }\end{array}$ & $\begin{array}{l}\text { Dicotómica } \\
1=\text { Sí } \\
0=\text { No }\end{array}$ \\
\hline
\end{tabular}

\section{RESULTADOS}

Para la determinación de la disposición a pagar por un sistema de recolección mejorado de los residuos sólidos domésticos se consideraron distintas variables de relevancia (Tabla 1), donde cada una de estas tiene un comportamiento particular y característico en la población objeto de estudio. La variable más significativa que explica la disposición a pagar del usuario es el nivel de educación. Del total de encuestados, el 10.99 \% indicaron que tienen un nivel de educación de 16 años (superior universitario). Asimismo, señalaron que están dispuestos a pagar por el sistema de recolección de residuos (Fig. 1). Se demuestra así la importancia de la formación educativa para la práctica ambiental en los RS, considerando los procesos que involucra la misma. Entonces, a medida que la población tiene un nivel educativo más alto, estaría dispuesta a pagar por contar con un sistema de recolección de residuos sólidos, al ser más conscientes de los beneficios.

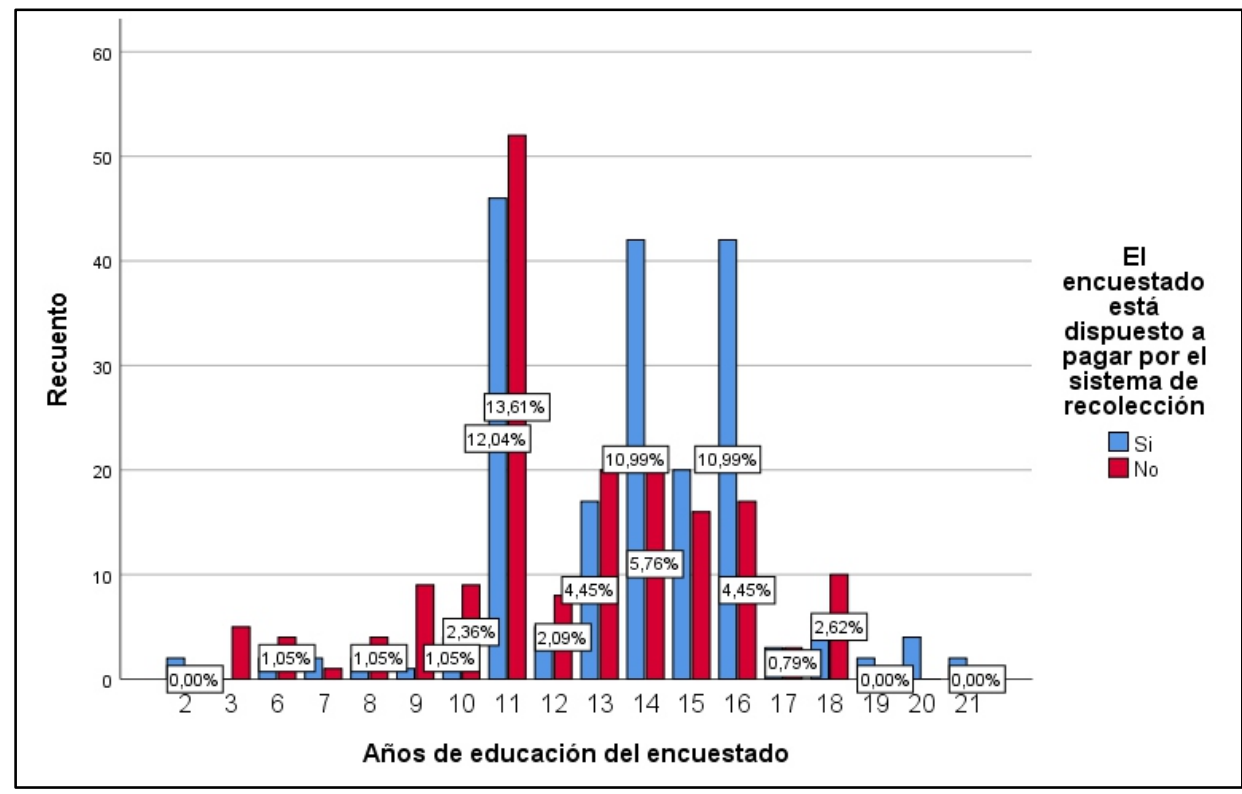

Figura 1. Disponibilidad a pagar por el sistema de recolección 
La variable "ética ambiental" también es considerada de importancia para la determinación de la disposición a pagar (Tabla 2), donde el encuestado considera que es importante la conservación del medio ambiente. El $51.57 \%$ de los encuestados consideran que tienen la disposición de pagar por un sistema de recolección mejorado de los residuos sólidos domésticos, en vista que es importante la conservación del medio ambiente. Por el contrario, el $41.10 \%$ no están dispuestos a pagar, pero que sí consideran que es importante la conservación del medio ambiente. Lo que se demuestra que, la influencia de esta variable puede representar un factor importante en la toma de decisiones de políticas públicas ambientales (Fig. 2).

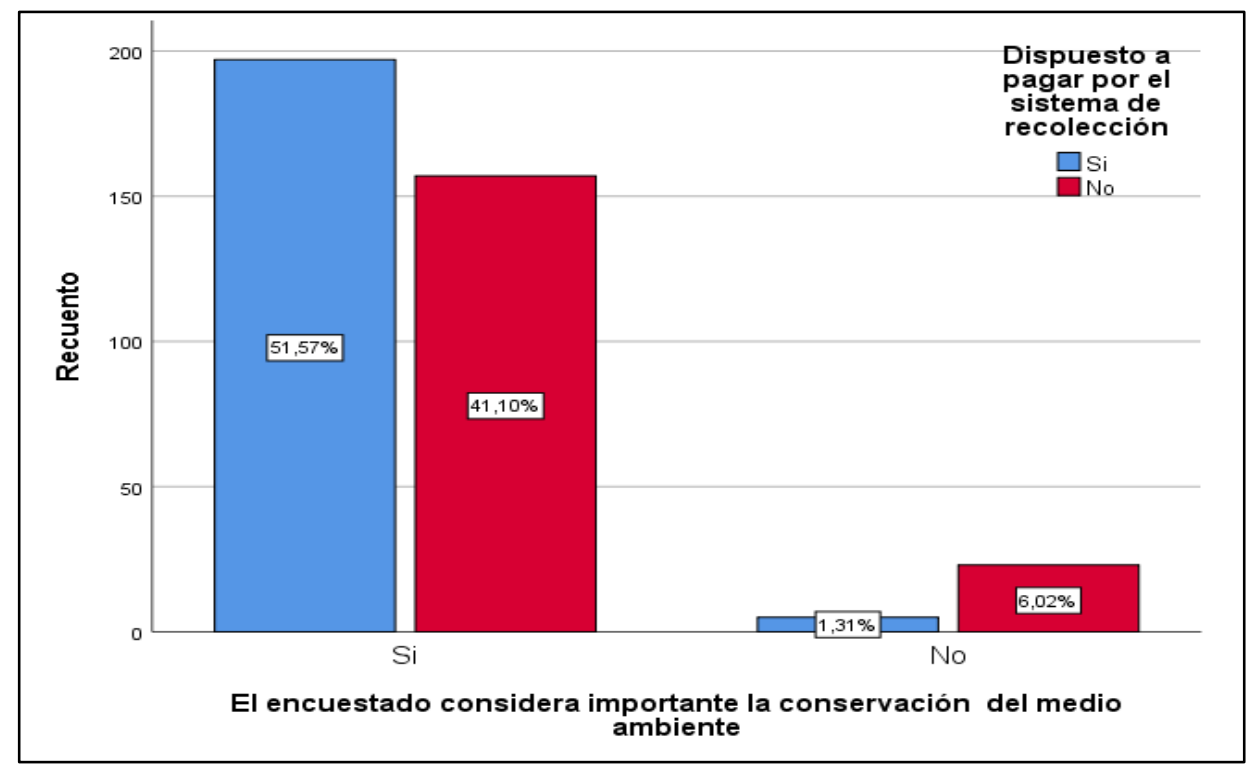

Figura 2. Importancia en la conservación del medio ambiente

Otra variable relevante es la "consciencia ambiental", donde el $27.29 \%$ de los encuestados considera que realiza acciones para evitar la contaminación y, además, tiene la disposición a pagar por el sistema de recolección de residuos sólidos. Por otro lado, el 13.61 \% señala que realiza acciones para evitar la contaminación, pero no están dispuestos a pagar. En contraste, con respecto a la población que no realiza acciones para evitar la contaminación, el 25.39 \% estaría dispuesto a pagar por el sistema de recolección. Sin embargo, el $33.51 \%$ no estaría dispuesto a realizar ese pago. Lo que refleja la actitud de las personas frente a la conservación del medio ambiente (Fig. 3).

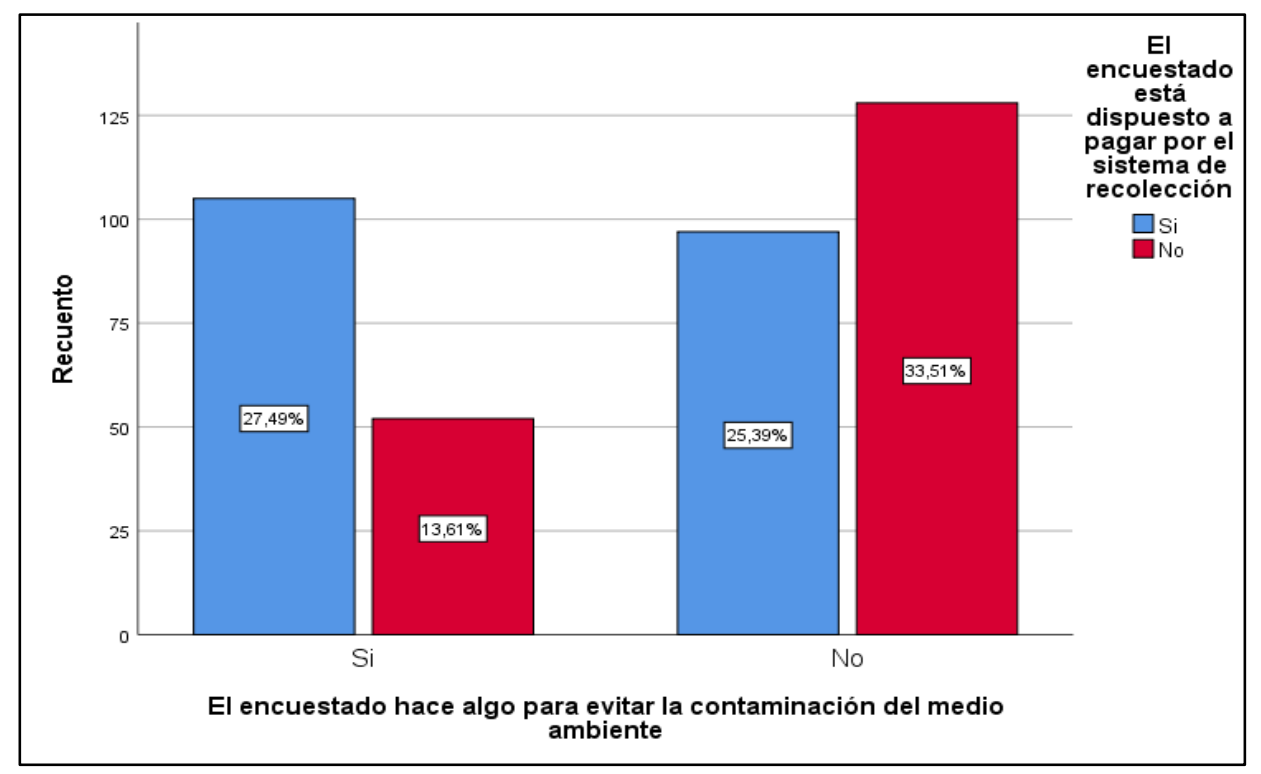

Figura 3. Consciencia Ambiental de la población 
Complementariamente a la importancia de la conservación del medio ambiente, esta se relaciona a la educación ambiental que tiene el ciudadano, demostrando la relevancia del medio ambiente sobre sus quehaceres. Esto se plasma en acciones o actividades que realiza el poblador para contribuir con ello. En este sentido, del total de los encuestados, el $41.10 \%$ indicaron que sí realizan alguna acción para evitar la contaminación del medio ambiente en su hogar. Las acciones que realizan son reciclar $(22.42 \%)$, separar la basura (19.59\%), además de otras acciones (6.96\%) (Fig. 4).

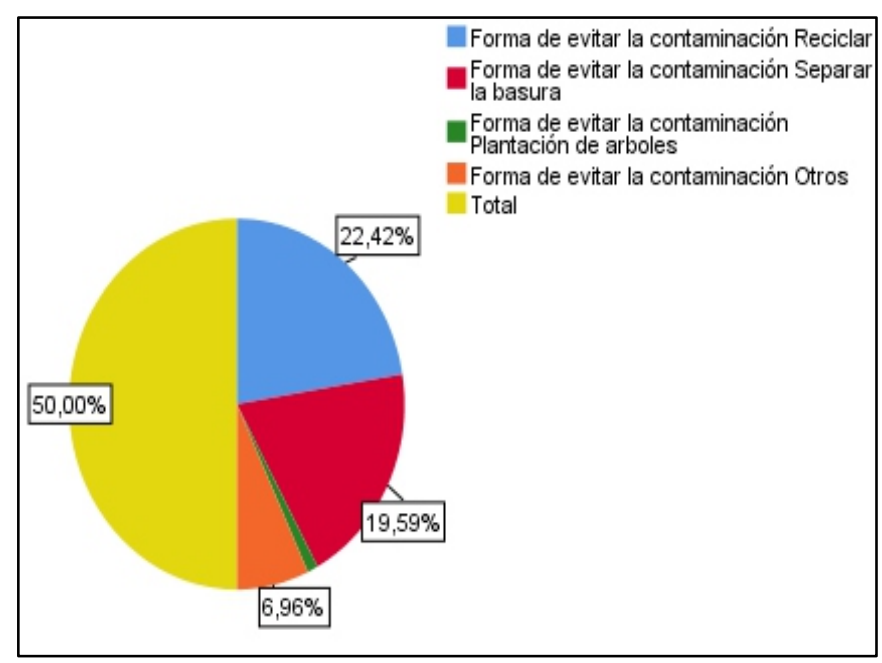

Figura 4. Formas de evitar la contaminación

En este sentido, cabe resaltar que la disposición a pagar por un sistema de recolección mejorado de los residuos sólidos domésticos es determinante. De ahí que, del total de la muestra de los 382 hogares encuestadas en la ciudad de Juliaca, se puede verificar que los montos más significativos a pagar son de S/. $2.00(15.84 \%), \mathrm{S} / .5 .00(37.13 \%)$ y S/. $10.00(16.83 \%)$. Este monto está sujeto al ingreso promedio del hogar y al nivel de información que se tiene sobre los efectos negativos que ocasionan los residuos sólidos domésticos. Sin embargo, solo un $16.83 \%$ y $37.13 \%$ de los encuestados está dispuesto a pagar una cantidad superior a los montos ya mencionados, los cuales oscilan entre $\mathrm{S} / 12.00$ y S/ 15.00 respectivamente (Fig. 5). Por lo cual, la disponibilidad de pago promedio es de S/ 5.36 mensuales, por un sistema de recolección de residuos sólidos domésticos.

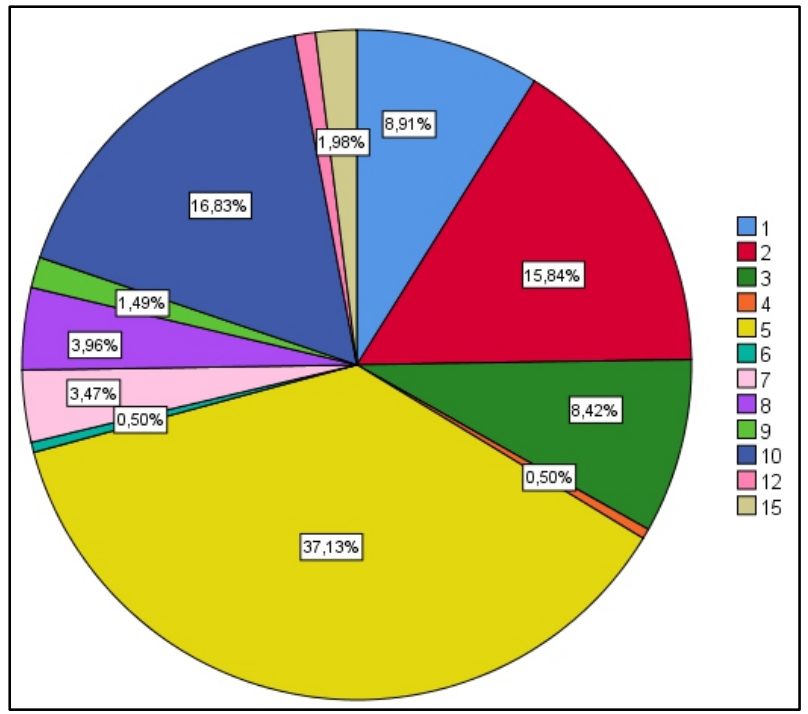

Figura 5. Cantidad monetaria que está dispuesto a pagar

Por otro lado, los hogares estarían dispuestos a realizar el pago en forma mensual. Asimismo, se han identificado variables significativas: el nivel educativo, ética ambiental y la conciencia ambiental de las personas (Tabla 2). 
.Por otro lado, los hogares estarían dispuestos a realizar el pago en forma mensual. Asimismo, se han identificado variables significativas: el nivel educativo, ética ambiental y la conciencia ambiental de las personas (Tabla 2).

Analizando lo señalado en el párrafo anterior, mientras las personas tengan un mayor grado de educación, estarían dispuestas a pagar por un sistema de recolección con el objetivo de mejorar su bienestar. Otra variable de importancia es la ética ambiental de las personas, lo cual guarda una relación positiva. Por ende, se podría decir que mientras más alto sea el nivel de ética ambiental, mayor será la disponibilidad de pago. Cabe recalcar que, en la presente investigación se trabajó con un nivel de confianza de $95 \%$.

Además, se investigaron de manera empírica los factores influyentes en la disposición a pagar por un sistema de recolección de residuos sólidos domésticos y también las variables significativas sobre el modelo utilizado. Para ello, se utilizó el modelo econométrico Probit. En este caso, las variables que son estadísticamente significativas para explicar la disposición a pagar son el nivel educativo, ética ambiental y conciencia ambiental, como se observa en la Tabla 2.

Tabla 2. Estimación del modelo Probit binomial

\begin{tabular}{|c|c|c|c|c|c|c|}
\hline \multirow[b]{4}{*}{ Log likelihood $=-236.02789$} & & & & & Number of obs & 382 \\
\hline & & & & & LR chi2(7) & 56.24 \\
\hline & & & & & Prob $>$ chi2 & $=0.0000$ \\
\hline & & & & & Pseudo R2 & 0.1065 \\
\hline disp pagar & Coef. & Std. Err. & $\mathrm{Z}$ & $\mathrm{P}>|\mathrm{z}|$ & \multicolumn{2}{|c|}{ [95\% Conf. Interval] } \\
\hline ingreso $_{\log }$ & 0.0466093 & 0.1250218 & 0.37 & 0.709 & -0.1984288 & 0.2916474 \\
\hline Gen & 0.0998368 & 0.1469619 & 0.68 & 0.497 & -0.1882031 & 0.3878768 \\
\hline edad & -0.0024818 & 0.0060686 & -0.41 & 0.683 & -0.0143761 & 0.0094125 \\
\hline$N$ & -0.0396281 & 0.0434407 & -0.91 & 0.362 & -0.1247703 & 0.0455142 \\
\hline educación & 0.081437 & 0.0246897 & 3.30 & 0.001 & 0.033046 & 0.129828 \\
\hline$E a$ & -0.5706758 & 0.1429566 & -3.99 & 0.000 & -0.8508657 & -0.290486 \\
\hline evit_contam & 1.221 .764 & 0.31352 & 3.90 & 0.000 & 0.6072763 & 1.836 .252 \\
\hline Cons & -133.422 & 1.012 .472 & -1.32 & 0.188 & -3.318 .629 & 0.6501895 \\
\hline
\end{tabular}

Se puede observar en la regresión presentada que el indicador de bondad de ajuste es 0.1065 . Esto quiere decir que aproximadamente el $10.65 \%$ de los cambios en la variable dependiente "sí está dispuesto o no a pagar" depende de las variables independientes. Entre las variables significativas presentadas en el modelo se encuentran "el nivel de educación", la cual guarda una relación positiva; y "la ética ambiental de las personas", representada por la importancia que le dan las personas a la conservación del medio ambiente, guardando una relación positiva con la variable dependiente. Por ende, mientras mayor sea el nivel de ética ambiental de las personas, mayor es la probabilidad de estar dispuestas a pagar por un sistema de recolección de residuos sólidos domésticos.

La variable "conciencia ambiental" es otra variable significativa que guarda una relación negativa. Es decir, la probabilidad de que una persona tenga una menor conciencia ambiental sobre la conservación del medio ambiente. Por ejemplo, cuando una persona no está realizando acciones para evitar contaminar el ambiente, tiene más posibilidades de aportar monetariamente por un sistema que ayude en la conservación del medio ambiente, como un sistema de recolección de residuos sólidos.

En la Tabla 3, las variables más relevantes del modelo Probit para cada uno de los coeficientes se explica de la siguiente manera:

- Si el ingreso per cápita del hogar (ingreso ${ }_{\log }$ ) incrementa en $\mathrm{S} / 1.00$, la probabilidad de pagar por la mejora en el manejo de residuos sólidos domésticos en la ciudad de Juliaca aumenta en un $1.82 \%$.

- Si la edad de la persona ( $e d a d$ ) incrementa en un (01) año, la probabilidad de pagar por la mejora en el manejo de residuos sólidos domésticos en la ciudad de Juliaca disminuye en un $0.098 \%$. 
- Si el nivel educativo del encuestado (educación) aumenta en un (01) año más de educación, la probabilidad de pagar por la mejora en el manejo de residuos sólidos domésticos en la ciudad de Juliaca incrementará en $3.25 \%$.

- Si el encuestado hace algo para evitar la contaminación del medio ambiente $(E a)$, la probabilidad de pagar por la mejora en el manejo de residuos sólidos domésticos en la ciudad de Juliaca disminuirá en $22.5 \%$.

- Si el encuestado considera importante la conservación del medio ambiente (evit_contam), la probabilidad de pagar por la mejora en el manejo de residuos sólidos domésticos en la ciudad de Juliaca incrementará en $41.9 \%$.

Tabla 3. Efectos marginales

\begin{tabular}{lcccc}
\hline $\begin{array}{l}\text { Marginal effects after probit } \\
\mathrm{Y}=\operatorname{Pr}(\text { disp_pagar) (predict) } \\
=0.0466093\end{array}$ & & & & \\
\hline \multicolumn{1}{c}{ disp_pagar } & dy/dx & Std. Err. & $\mathrm{Z}$ & $\mathrm{P}>|\mathrm{z}|$ \\
\hline ingreso $_{\log }$ & 0.0185524 & 0.04976 & 0.37 & 0.709 \\
Gen & 0.039712 & 0.05839 & 0.68 & 0.496 \\
edad & -0.0009878 & 0.00242 & -0.41 & 0.683 \\
$N$ & -0.0157736 & 0.01729 & -0.91 & 0.362 \\
educación & 0.0324153 & 0.00983 & 3.30 & 0.001 \\
Ea & -0.2271526 & 0.05689 & -3.99 & 0.000 \\
evit_contam & 0.4188522 & 0.07449 & 3.62 & 0.000 \\
\hline
\end{tabular}

\section{DISCUSIÓN}

A partir de la presente investigación se conoce la disponibilidad de pagar por un sistema adecuado de recolección de residuos sólidos domésticos en la ciudad de Juliaca. De la cual, el $51.57 \%$ de los encuestados estarían dispuestos a pagar por dicho sistema, esto debido a la preocupación del problema existente. Sin embargo, el $41.10 \%$ de los encuestados no estarían dispuestos a pagar, señalando que es responsabilidad de la municipalidad. Otro motivo es no contar con los suficientes recursos económicos para pagar, siendo esto concordante a lo planteado por Nuñez \& Tenorio (2016) y por Ibáñez \& Corroppoli (2002).

En el modelo expuesto, se evidencia que existen variables que no han alcanzado el nivel de confianza esperado, es decir, menor al $5 \%$. Por tal motivo, se ha obtenido un $R^{2}$ relativamente bajo. Estas variables son: el ingreso per cápita, la edad, el género, y el número de integrantes del hogar; ya que no producen ningún tipo de efecto sobre la variable endógena (Saidón, 2012). Asimismo, las variables independientes consideradas en el modelo obtenido explican en un $10.65 \%$ a la variable dependiente, el mismo que muestra ser un modelo conveniente para este tipo de investigaciones con esta metodología (Mercado et al., 2009). Además, las tres variables significativas que explican el modelo son: el nivel de educación con un coeficiente de 0.081 , la ética ambiental con un coeficiente de 1.22 y la conciencia ambiental con un coeficiente de -0.57. Relativamente, los coeficientes de las variables significativas son bajos, comparándolos con los determinados por Farreras \& Lauro (2017) y Pérez (2018), debido a la variedad de información, la cual no garantiza la veracidad de los resultados.

Por lo que, al igual que con la investigación de Pérez (2018) y Castro (2010), se coincide con los resultados obtenidos. Los encuestados determinan que el problema de los residuos sólidos en la ciudad de Juliaca no es tomada en cuenta por las autoridades responsables, en vista que el equipo técnico que respalda a las actuales gestiones no valora la importancia que tiene el sistema de recolección de los residuos sólidos. En este sentido, la disposición promedio a pagar es de S/. 5.36, por un sistema de recolección de residuos sólidos domésticos, donde la variable más resaltante es la conciencia ambiental que tienen las personas sobre el medio ambiente. Dicha variable guarda una relación positiva con la disposición a pagar, es decir, cuando una persona 
toma conciencia ambiental, entonces mayor será la disponibilidad de pago (Mamani, 2019).

En el caso del estudio realizado por Chambilla (2015), en la ciudad de Puno, se determinó que el $55.90 \%$ de la población de Puno está dispuesta a pagar el monto en promedio de S/. 4.45 (mensualmente) por cada hogar urbano, por una mejora en el manejo de los residuos sólidos, concordando con lo establecido por Farreras \& Lauro (2017). Cada vez que las personas son más conscientes de la importancia del medio ambiente, estarían dispuestas a aportar para tener un sistema de recojo de residuos que conlleve al posterior proceso para una buena disposición final.

Además, en el caso de estudio realizado por Romero (2017), en el distrito de Caracoto de la región de Puno, la disposición a pagar (DAP) hallada fue de $\mathrm{S} / 1.695$ por un adecuado manejo de la producción per-cápita de residuos sólidos, donde el $81 \%$ de la población estudiada estaban de acuerdo en contribuir de manera económica para la mejora del escenario actual. En el caso de Rojas (2012), quien aplicó la misma metodología para la ciudad de Puno, la disponibilidad de pago de la población fue de S/ 13.07 mensuales por familia por la mejora en el manejo de los residuos sólidos urbanos, donde el $62.56 \%$ de los entrevistados estaba dispuesto a pagar.

Contrastando con estudios realizados a nivel internacional, Ibarrarán \& Cuevas (2013) consideran en su investigación, que la población objeto de estudio estuvo dispuesta a pagar un valor de \$. 4.2 millones de pesos (612 945.06 soles) anuales que equivale a $\$ .18 .47$ pesos (2.70 soles) mensuales por vivienda, mostrando que tienen el interés de alcanzar el escenario deseado.

Finalmente, cabe mencionar que, sobre las limitaciones de la presente investigación, estas radican y se generaron debido a la deficiencia que hubo en la realización de las encuestas realizadas a personas mayores, representante de un hogar, en la ciudad de Juliaca. Muchas de estas se encuentran en el desarrollo de las actividades comerciales (Se encuentran en ferias), laborando en alguna institución pública o privada o viajando por motivos de negocios; lo cual limita el proceso de determinación del valor próximo al valor ambiental (Galarza \& Gómez, 2005). Esto, considerando la importancia del desarrollo de la gestión ambiental de los RS, en vista que el proceso de segregación, tratamiento, procesamiento y disposición final es importante, a fin de lograr el buen aprovechamiento de los residuos con diferentes fines posteriores (Cotler et al., 2007).

Por tal motivo, se decidió analizar las variables influyentes en la determinación de la disponibilidad promedio a pagar para tener un mejor manejo de los residuos sólidos en la ciudad de Juliaca. Por lo tanto, se obtuvieron resultados cuantitativos con el uso de las encuestas y análisis estadístico, siendo estos muy importantes para la toma de decisiones y aplicación de las posibles políticas públicas medioambientales (Roldan, 2016; Pareja, Villalba, Calderón \& Sánchez, 2015).

\section{CONCLUSIONES}

El $51.75 \%$ de los encuestados de la población de Juliaca están dispuestos a pagar por una mejora en el sistema de recojo de residuos sólidos domésticos con un monto promedio de $\mathrm{S} / 5.36$ mensuales. Mientras que el $41.10 \%$ de los encuestados no estaría dispuesto a pagar debido a que dicha medida es responsabilidad de la municipalidad, además, indican que no tienen los suficientes recursos económicos para pagar.

Las variables significativas o más relevantes que explican la disposición a pagar por un sistema de recolección mejorado de los residuos sólidos domésticos son el nivel de educación, el cual guarda una relación positiva con la disposición a pagar en un porcentaje de $3.24 \%$; el nivel de ética ambiental de las personas, demostrado en la importancia que le dan las personas a la conservación del medio ambiente, lo que guarda una relación positiva con la variable dependiente en un porcentaje de $41.8 \%$; y la conciencia ambiental, que guarda una relación negativa en un $22.5 \%$.

\section{AGRADECIMIENTO}

A la Agencia Chilena de Cooperación Internacional para el Desarrollo (AGCID), por el apoyo hacia el fomento de la investigación social y ambiental en América Latina y el Caribe. A las autoridades de la Universidad Nacional del Altiplano, Facultad de Ingeniería Económica y a la Universidad Nacional de Moquegua, quienes nos motivaron en el diseño del presente trabajo de investigación. 


\section{REFERENCIAS}

Armijo de Vega, C., Puma Chávez, A., \& Ojeda Benítez, S. (2012). El conocimiento de los habitantes de una ciudad mexicana sobre el problema de la basura. Revista internacional de contaminación ambiental, 28, 29-37.

Azqueta Oyarzun, D. (1994). Valoración económica de la calidad ambiental. McGraw-Hill. Madrid, España.

Castro Sobarzo, P. (2010). Disposición a pagar por la incorporación de un sistema de reciclaje para los residuos sólidos domiciliarios en la comunidad de Puerto Montt, Región de Lagos (tesis pregrado) Universidad Austral de Chile. Puerto Montt, Chile.

Chambilla Chachaque, J. F. (2015). Valoración económica por la mejora en el manejo de residuos solidos urbanos en la ciudad de Puno, año 2012 (Tesis de pregrado). Recuperado de http://repositorio.unap.edu.pe/handle/U NAP/3292

Cotler, H., Sotelo, E., Domínguez, J., Zorrilla, M., Cortina, S., \& Quiñones, L. (2007). La conservación de suelos: un asunto de interés público. Gaceta ecológica, (83), 5-71.

Farreras González, V. I., \& Lauro, C. (2017). Valoración económica de los efectos de la contaminación por vertido de residuos sólidos urbanos: El caso del aglomerado urbano del Gran Mendoza, Argentina. Revista Gestión y Ambiente, 211-227.

Fernández Collado, C., Baptista Lucio, P., \& Hernández Sampieri, R. (2006). Metodología de la investigación. Editorial McGraw Hill.

Galarza, E., \& Gómez de Zea, R. (2005). Valorización económica de servicios ambientales: el caso de Pachacamac, Lurín. Universidad del Pacífico.

Girón, L. E. (2017). Econometría aplicada usando stata 13. Sello Editorial Javeriano-Pontificia Universidad Javeriana, Cali.

Guzmán Chávez, M., \& Macías Manzanares, C. H. (2012). El manejo de los residuos sólidos municipales: un enfoque antropológico. El caso de San Luis
Potosí, México. Estudios sociales (Hermosillo, Son.), 20(39), 235-262.

Huacani Sucasaca, Y., \& Mamani Mamani, J. (2016). Valoración ambiental del reciclado de residuos sólidos en el caso de Juliaca.

Huamán Salhuana, Milena M. L. (2019). Contaminantes del ecosistema del lago Titicaca de la región Puno y la gestión ambiental del turismo (Tesis de pregrado). Recuperado de http://repositorio.usmp.edu.pe/handle/us $\mathrm{mp} / 4825$

Huamani Montesinos, C. (2017). Análisis socieconómico y ambiental del reaprovechamiento y disposición final de los residuos sólidos en la ciudad de Juliaca, San Román-Puno (Tesis de pregrado). Recuperado de http://repositorio.unap.edu.pe/handle/U NAP/6294

Huamaní Montesinos, C., Tudela Mamani, J. W., \& Huamaní Peralta, A. (2020). Gestión de residuos sólidos de la ciudad de Juliaca-Puno-Perú. Revista de Investigaciones Altoandinas, 22(1), 106115.

Ibáñez, J. R., \& Corroppoli, M. D. (2002). Valorización de residuos sólidos urbanos. Descargado de: http://www. bvsde. paho. org/bvsacd/cd48/valorizaresiduos. pdf Basagoitia García, JA.

Ibarrarán Viniegra, M. E., Ivan, I. C., \& Cuevas, E. M. (2003). Valoración económica del impacto ambiental del manejo de residuos solidos municipales, estudio de caso. Gaceta Ecológica, 69-82.

Lacort, M. O. (2014). Estadística Descriptiva e Inferencial-Esquemas de Teoría y Problemas Resueltos. Lulu. com.

Mamani, J. C. Q. (2019). Factores determinantes de la percepción pública sobre la contaminación atmosférica urbana de la ciudad de Juliaca, 2019. ÑawparisunRevista de Investigación Científica, 2(1).

Mamani, J. C. Q. (2019). Disposición a pagar por el sistema de recolección de los residuos sólidos domesticos en la ciudad de Juliaca, 2019. Semestre Económico, 8(2), 67-99.

Mercado, A., López, C. R., Flores, A., Giner, F., Graizbord, B., Lorea, A., \& Schatán, C. (2009). Desarrollo de las estadísticas del medio ambiente: planteamientos $\mathrm{y}$ 
conclusiones. Estudios demográficos y urbanos, 24(3), 697-727.

Nuñez Valladolid, G. A., \& Tenorio Villalobos, J. M. (2016). Determinantes de la disponibilidad a pagar para la reducción de residuos sólidos en el distrito de José Leonardo Ortiz.

Pareja, F. A., Villalba, L., Calderón, F., \& Sánchez, R. (2015). Propuestas para el manejo integrado de los residuos sólidos en el área metropolitana de Caracas (AMC). Politeia, 38(55), 1-31.

Pérez Alcatruz, M. I. (2018). Evaluacíon técnicaeconómica de la implementación de gestión de residuos solidos domicialiarios con un enfoque de basura cero en la comna de Recoleta.

Pérez, G. B. (2015). La gestión de los residuos sólidos: un reto para los gobiernos locales. Sociedad y Ambiente, 1(7), 72 98.

Quilla Ordoño, C. R. (2017). Valoración económica del tratamiento y gestión del manejo de los residuos sólidos urbanos en la ciudad de Huancané (Tesis de pregrado). Recuperado de http://repositorio.unap.edu.pe/handle/U NAP/5090

Rodriguez San Roman, C. M. (2017). Optimización del dimensionamiento de un relleno sanitario de residuos sólidos municipales para la ciudad de Juliaca 2015 (Tesis de pregrado). Recuperado de http://repositorio.uancv.edu.pe/handle/U ANCV/3018

Rojas Mamani, J. S. (2012). Disponibilidad a pagar por la mejora en el manejo de los residuos sólidos urbanos en la ciudad de
Puno, 2011 (Tesis de maestría).

Recuperado de http://repositorio.unap.edu.pe/handle/U $\mathrm{NAP} / 316$

Roldan Villalobos, R. I. (2016). Valoración económica ambiental de la recolección selectiva y tratamiento de los desechos sólidos. Caso de estudio: Cantón El Faro del Municipio de Comasagua.

Romero Kana, A. (2017). Disposición a pagar para el adecuado manejo de la producción percápita de residuos sólidos en el distrito de Caracoto - Puno (Tesis de pregrado). Recuperado de http://repositorio.unap.edu.pe/handle/U $\mathrm{NAP} / 7715$

Saidón, M. (2012). Valoración ambiental del reciclado de residuos:. Economía, XXXVII, 33-53.

Tudela Mamani, J. W., Martínez Damián, M. A., Valdivia Alcalá, R., Portillo Vázquez, M., \& Romo Lozano, J. L. (2009). Modelos de elección discreta en la valoración económica de áreas naturales protegidas. Revista mexicana de economía agrícola y de los recursos naturales.

Valdivia Alcalá, R., Abelino Torres, G., López Santiago, M. A., \& Zavala Pineda, M. J. (2012). Valoración económica del reciclaje de desechos urbanos. Revista Chapingo Serie Ciencias Forestales y del Ambiente.

Vargas González, A. P., \& Cortés, G. (2018). International experiences of sustainable use of disposable paper vessel. Producción+ Limpia, 13(2), 37-54. 